\title{
Tunable linear fiber laser cavity based on a twisted mechanical long period grating
}

\author{
E. Huerta-Mascotte ${ }^{a}$, J. M. Estudillo-Ayala ${ }^{b, *}$, R.I. Mata-Chávez ${ }^{a}$, D. Jáuregui-Vázquez ${ }^{a}$, \\ J.M. Sierra-Hernández ${ }^{a}$, J.A. Montenegro-Orenday ${ }^{b}$, H.J. Estrada-García ${ }^{b}$, and R. Rojas-Laguna ${ }^{b}$ \\ ${ }^{a}$ Departamento de Estudios Multidisciplinarios, División de Ingenierías Campus Irapuato-Salamanca, \\ Universidad de Guanajuato, Av. Universidad s/n, Col. Yacatitas, Yuriría, Gto., 38940, México. \\ ${ }^{b}$ Departamento de Electrónica, División de Ingenierías Campus Irapuato-Salamanca, Universidad de Guanajuato, \\ Carretera Salamanca-Valle de Santiago Km 3.5+1.8 Km, Salamanca, Gto., 3688, México. \\ e-mai: julian@ugto.mx
}

Received 23 April 2018; accepted 27 June 2018

\begin{abstract}
The present manuscript shows linear cavity fiber laser experimental analysis. The fiber laser arrangement, uses an all fiber mechanical long period grating (MLPG) to control single and dual laser emission. Here, single laser emission centered at $1562 \mathrm{~nm}$ with a signal to noise ratio of $27 \mathrm{~dB}$ and a linewidth less than $0.1 \mathrm{~nm}$ is obtained and tuned from $1562 \mathrm{~nm}$ to $1546 \mathrm{~nm}$, by twisting a conventional section of single mode fiber that was set into the MLPG. Thus, when the twist is around $120^{\circ}$ dual emission is obtained with centered wavelengths at 1533 $\mathrm{nm}$ and $1546 \mathrm{~nm}$, these emissions can be switched between them when the twist is incremented and the peak centered at $1546 \mathrm{~nm}$ is tuned in backward direction. The proposed laser offers wavelength and power stability with minimal variations of $0.5 \mathrm{~dB}$ and $0.5 \mathrm{~nm}$ respectively. This laser can be applied in several fields such as sensing, optical signal analysis and, optical communications.
\end{abstract}

Keywords: Mechanical long-period fiber gratings; twisted fiber optics; tunable linear fiber laser.

PACS: 42.55.-f; 42.55.Wd; 42.81.-i.

\section{Introduction}

Continuous and pulsed wave linear fiber laser cavities have been attractive for researchers during long period of time [1-3], owing to its several advantages such as: compactness, minimal operation elements and stability. Moreover numerous works deals to improve tunability or switch issues, as a result several arrangements based on Fabry-Perot filters [4-12], Mach-Zehnder interferometer [13-16], losses effect in gain medium [17], mechanical long period grating (MLPG) [18-20], Sagnac interferometers [21-23] and fiber Bragg grating (FBG) $[24,25]$ have been reported. One of the most attracted fiber filter used in fiber laser arrangements are MLPG's, indeed its wide tunable range by twist effect have been widely demonstrated [26,27], moreover this device offers simple design and low cost implementation as well as multiple sensor applications such as: temperature, refractive index and strain. It is important to point out that several long period gratings, fabricated by different techniques have been proposed as a fiber laser filter devices [28-30]. However, the period and length of these long period gratings can not be adjusted; as a result, physical change like curvature, twist, refractive index environment change or temperature must to be applied over a fragile structure in order to excite tuned or switched effect. In the presented experimental analysis, an adjustable MLPG was set into the linear fiber laser cavity acting as a fiber filter device. Here, a short length of conventional single mode fiber was twisted before to set into the MLPG, as a consequence the tuned and switched effect can be controlled; due to the gain-losses profile is modified into the laser cavity.

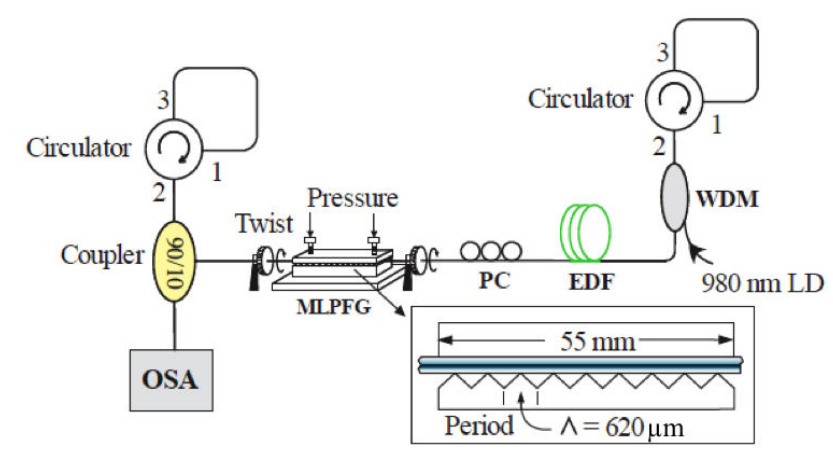

FIGURE 1. Schematic configuration of linear fiber laser arrangement (inset) sketch mechanical long period grating.

In Fig. 1 the schematic configuration of the switchable and tunable linear fiber laser cavity is presented. This fiber laser arrangement is based on four main elements: two loop reflectors based on an optical fiber circulator, a gain medium, provided by $3.5 \mathrm{~m}$ of Erbium-Doped Fiber LIEKKI ${ }^{\mathrm{TM}}$ Er168/125 (EDF) and an all fiber MLPG as a cavity loses device which allow us to tuned and switch the fiber laser. Using a 980/1550 nm optical fiber wavelength division multiplexer (WDM), the laser diode (QFBGLD-980-200) with a centered wavelength operation at $980 \mathrm{~nm}$ and maximal pump power of $200 \mathrm{~mW}$ was coupled to the cavity. This laser pump is directed to the EDF, as a result, amplified spontaneous emission (ASE) is generated and guided to the MLPG, (the principle operation will be detailed below) in this point the cavity loses are modified. The light continues to optical fiber coupler (90/10) which allows us to monitoring the laser output by the $10 \%$ coupler port connected to the Optical Spec- 
trum Analyzer (OSA) Yokogawa, AQ6370B. The rest of the light $(90 \%)$ continues to the first optical fiber loop reflector, which reflect the light to second optical fiber reflector set at the $1550 \mathrm{~nm}$ WDM port, as a consequence ASE oscillation is generated into the cavity and single line emission is obtained in the laser output. In order to have gain and loses equilibrium a polarization controller (PC) was set between EDF and the MLPG.

\section{Results and Discussion}

The linear fiber laser proposed is tuned by an all fiber mechanical long period grating. Here, a small length of conventional single mode fiber (SMF28) was fixed between two metallic plates, one is totally flat and another one is v-grooved (see Fig. 1 inset), with a period of $620 \mu \mathrm{m}$ and a length around $55 \mathrm{~mm}$. Using the screws showed in Fig. 1 inset, lateral force is applied over the flat plate, this force can be estimated using a digital torque meter and the relations between torque and force described by [31]. The lateral force applied over the MLPG generated an interaction between fundamental core mode and the high order cladding modes, as a consequence, attenuation band centered at specific wavelength is obtained. The phase matching condition is satisfied by:

$$
\lambda_{n}=\left(n_{\text {eff core }}-n_{\text {eff clad }(n)}\right) \Lambda
$$

where, $n_{\text {eff core }}$ is the core mode effective refractive index, $n_{\text {eff clad }(n)}$ is the effective refractive index of the high order cladding mode exited and $\Lambda$ is the period grating. Moreover, before adjusting the load to $22 \mathrm{lb}$ for each rotation of the fiber, the SMF28 was turned 15 degrees clockwise by a fiber optic support rotator, and we obtained the red line see Fig. 2, we immediately removed the load and turned the SMF28 15 degrees more by adjusting the load again and the blue line was obtained and so on by rotating the fiber and adjusting the load until obtaining Fig. 2, as a result wide tunable range can be obtained. This has to do with the excitation of high order

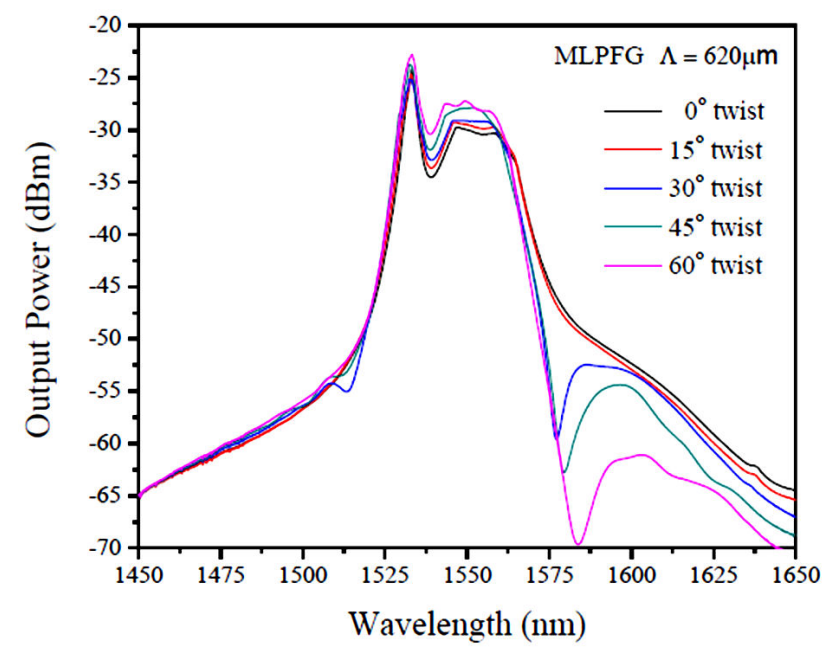

FIGURE 2. Mechanical long period grating power spectrum.

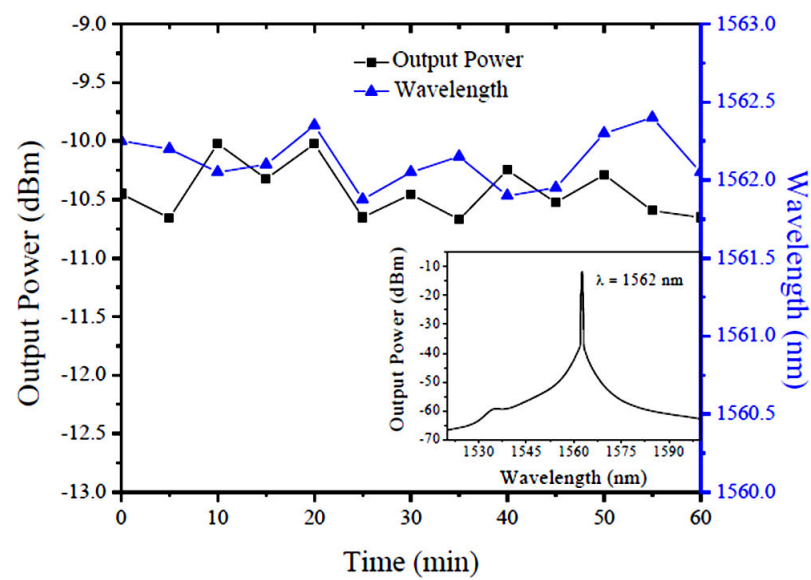

FIGURE 3. Power and wavelength stability analysis.

cladding mode promoted by the birefringence change generated when the SMF28 is twisted. According to Fig. 2, the notches observed in the MLPG spectrum can be tuned to longer wavelength when the fiber optic rotator is operated at the clockwise direction with constant lateral force $(22 \mathrm{lb}$ ) as can be appreciated. The MLPG will modify the gain spectrum into the linear laser cavity, as a result tuned and switchable effect can be expected.

The linear laser experimental analysis was carryout at room temperature using $130 \mathrm{~mW}$ of pump laser diode power. The linear laser cavity generates a single line emission centered at $1562 \mathrm{~nm}\left(\lambda_{p_{1}}\right)$ with a side mode suppression ratio (SMSR) of $27 \mathrm{~dB}$ and a linewidth less than $0.1 \mathrm{~nm}$ and maximal peak power of $-10 \mathrm{dBm}$ (see Fig. 3. inset). The output power spectrum was monitored each 5 minutes during an hour; as can be appreciated in Fig. 3 and minimal wavelength and power variations of $0.5 \mathrm{~nm}$ and $0.5 \mathrm{dBm}$ were observed respectively.

As was mentioned above the MLPG was operated with a constant lateral force around $22 \mathrm{lb}$ in both screws; in order to obtain tunable effect, the conventional SMF28 length set into

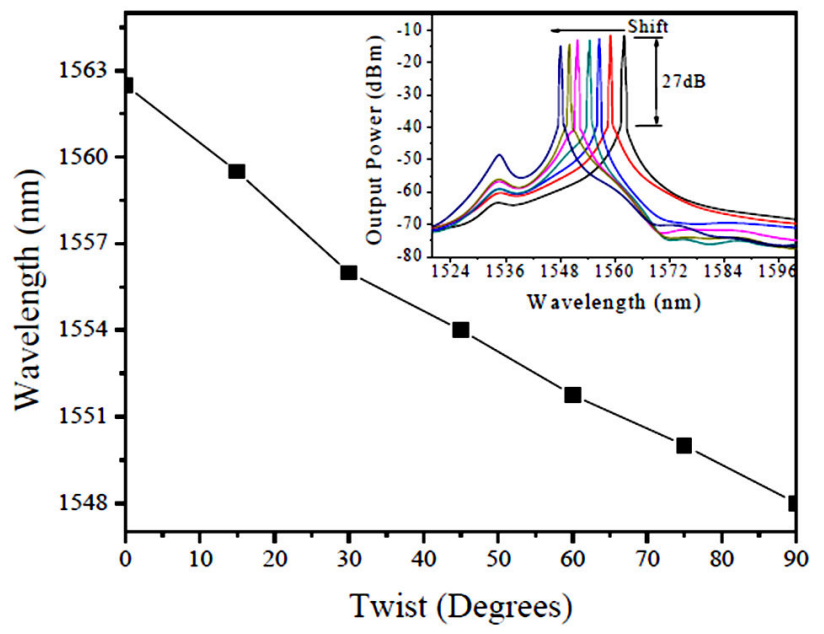

FIGURE 4. Tunable linear laser effect when the twist is applied into the MLPG. 


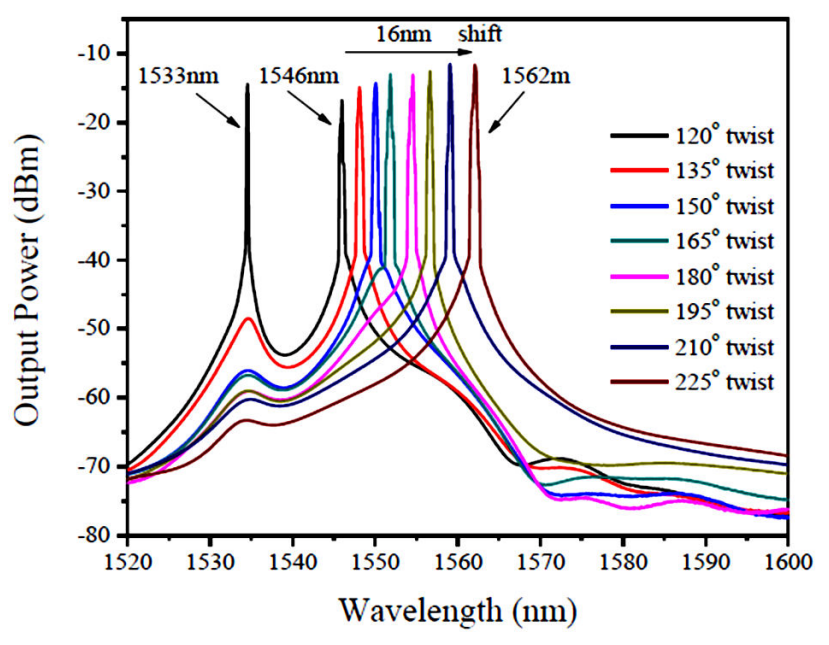

FIGURE 5. Dual and redshift laser emission.

the MLPG was twisted each $15^{\circ}$ from $0^{\circ}$ to $90^{\circ}$ and the output was recording. As can be observed in Fig. 4, lasing emission $\lambda_{p_{1}}$ was tuned from $1562 \mathrm{~nm}$ to $1548 \mathrm{~nm}$, with a total range of $16 \mathrm{~nm}$ and minimal spacing mode achieved around $1 \mathrm{~nm}$. Moreover when the rotation value is $120^{\circ}$, dual laser emission is obtained (see Fig. 5), and lasing mode centered at $\lambda_{p_{2}} 1546 \mathrm{~nm}$ and $\lambda_{p_{3}} 1533 \mathrm{~nm}$ were achieved with a SMSR of $27 \mathrm{~dB}$ and linewidth less than $0.1 \mathrm{~nm}$ were obtained. Here, the spacing mode between the dual laser emissions is around $12 \mathrm{~nm}$.

When the fiber is twisted circular birefringence is induced in the SMF28 [32], as a result, the excitation of high order cladding modes in the MLPG is promoted and wavelengthdependence losses is generated, which modifies the gain-loss medium profile into the linear laser cavity. As the twist increase the losses and high order mode energy also increase. Nevertheless, when the twist is around $120^{\circ}$ the birefringence generated a significant high order mode energy which provide the laser line centered at $1533 \mathrm{~nm}$, and the MLGP promotes the peak centered at $1546 \mathrm{~nm}$, obtaining dual laser emission as can be observed in Fig. 5. As the twist is modified around $102^{\circ}$ lasing modal competition can be expected between the dual laser emissions, as a consequence switchable effect is obtained between the lasing modes. Besides, when the twist is increase from $120^{\circ}$, redshift can be appreciated in Fig. 5.

\section{Summary}

In conclusion, stable and compact linear fiber laser cavity was experimentally demonstrated. Using a mechanical long period grating (MLPG) with $55 \mathrm{~mm}$ length and V-grooved period around $620 \mu \mathrm{m}$ set into the linear laser cavity, a single line emission centered at $1562 \mathrm{~nm}$ can be tuned to $1546 \mathrm{~nm}$, by applying constant lateral force $(22 \mathrm{lb}$ ) and by twisting conventional single mode fiber each $15^{\circ}$ from $0^{\circ}$ to $120^{\circ}$. Besides, when $120^{\circ}$ twist is applied dual laser emission is obtained, when the twist is incremented switchable and redshift effect were observed between these lasing modes. The laser offers a tuned range of $16 \mathrm{~nm}$ for single emission and it gets double wavelength and switchable for 120 degrees of twist.

\section{Acknowledgments}

E. Huerta-Mascotte recognized the support provided by Universidad Tecnológica del Suroeste de Guanajuato. This work was supported by DAIP Project "108/Investigación de los efectos de luz polarizada en los sistemas láseres de fibra óptica sintonizables" and CONACyT project CB2016286916.
1. X. Dong, N. Q. Ngo, and P. Shum, Opt. Express 11 (2003) 1689.

2. J. Yang, Opt. and Laser Technol. 34 (2002) 599

3. Y. Yu, C. Xiangfei, D. Yitang, and X. Shizhong, IEEE Photon. Technol. Lett. 18 (2006) 187.

4. C. H. Yeh, C. W. Chow, Y. F. Wu, F. Y. Shih, C. H. Wang, and S. Chi, Opt. Fiber Technol. 15 (2009) 344.

5. S. Pan, C. Lou, and Y. Gao, Opt. Express 14 (2006) 1113.

6. A. Zhou, B. Qin, Z. Zhu, Y. Zhang, Z. Liu, J. Yang, and L. Yuan, Opt. Lett. 39 (2014) 5267

7. X. Tan, Y. Geng, X. Li, R. Gao, and Z. Yin, Appl. Opt. 52 (2013) 8195.

8. S. Liu et al., Opt. Lett. 39 (2014) 2121

9. M. S. Ferreira, J. Bierlich, S. Unger, K. Schuster, J. L. Santos, and O. Frazao, IEEE Photon. Technol. Lett. 25 (2013) 1593

10. D. -W. Duan, Y.-J. Rao, Y.-S. Hou, and T. Zhu, Appl. Opt. 51 (2012) 1033.
11. D. Jáuregui-Vázquez et al., IEEE Photon. Journal 7 (2015).

12. D. Jáuregui-Vázquez et al., Opt. Commun. 308 (2013) 289.

13. J. M. Sierra-Hernández et al., Laser Phys. 23 (2013) 125103.

14. W. G. Chen et al., Laser Phys. 19 (2009) 2115.

15. A.-P. Luo, Z.-C. Luo, and W.-C. Xu IEEE Photon. Journal 3 (2011) 197.

16. J. M. Sierra-Hernández et al., Laser Phys. 23 (2013) 055105.

17. Seyed Rasoul Hosseinil, Mohammad Razaghil, Narottam Das, IEEE NUSOD PP. (2010) 43.

18. Xiaojun zhou, Chengjin Chen, Lan Lan, Zhiyao Zhang, Yong Liu, IEEE Photon. and Optoelectronics, (Symp. 2009).

19. M. Ichikawa, S. Araki and H. Laser Phys. Lett. 10 (2013) 025101

20. Hajime Sakata, Hitoshi Yoshimi, Yuki Otake, Opt. Commun. 282 (2009) 1179 
21. C. Ouyang et al., IEEE Quant. Electron. Journal 47 (2011) 198.

22. B. Ibarra-Escamilla et al., J. Eur. Opt. Soc. 3 (2008) 08036.

23. D. Chen, S. Qin, L. Shen, H. Chi, and S. He, Microwave and Opt. Technol. Lett. 48 (2006) 2416.

24. Dae Seung Moon, Un-Chul Paek, and Youngjoo, Opt. Express 13 (2005) 5615.

25. M. A. González-Reyna et al., IEEE Photon. Technol. Lett. 27 (2015) 1141-1144.

26. S. Anitha, V. P. Nair, K. Sudeep, and Hubert Appl. Opt. Soc. Am. 54 (2015) 2007-2010.
27. O.V. Ivanov, Opt. Commun. 232 (2004) 159-166.

28. L. L. Shi, T. Zhu, F. Y. Chen, M. Deng, and W. Huang, Laser Physics 22.3 (2012) 575-578.

29. C.Y. Lin, L.A. Wang, IEEE Photon. Technol. Lett. 13 (2001) 1561-1563.

30. Richard G. Budynas and J. Keith Nisbett, Shigley's mechanical engineering design edit. (McGraw-Hill Ninth edition 2011).

31. J.M. Estudillo-Ayala et al., Opt. and Lasers in Eng. 39 (2003) 635-643. 